\title{
Dynamics of Birth Spacing in Pakistan
}

\section{Framurz Khan Kiani and Samina Nazli*}

In recent years the study of birth spacing has emerged as an area of demographic interest. This is because of its close linkage with the timing and tempo of fertility relative to other measures. At the same time, marital fertility in Pakistan and the duration of breastfeeding have shown a decline (Khan 1985). Thus, the study of birth spacing becomes an important area for examining changes in the components of fertility. The paper's major objectives are to study:

(1) Changes in spacing between births if any.

(2) Differentials in birth spacing by residence in major 'urban', 'other urban' and 'rural' areas to see if there is a gradation in birth spacing by levels of urbanization.

(3) And differentials in birth spacing by age at marriage, urbanization, education, work pattern and contraceptive use.

This study has analysed data of the Population Labour Force and Migration (PLM) Survey 1979-80. Birth itervals were computed using information drawn from birth histories giving birth order and date of birth (Irfan 1981). The life table technique was considered appropriate for analysing birth spacing because certain biases like heaping, selectivity and censoring can be controlled. Only the most recent birth spacing experience amongst females in the sample was included to avoid problems of recall amongst older women. The study is based on births during the 10 years from 1967 to 1977.

To analyse trends in birth spacing, the median intervals from the Population Labour Force and Migration (PLM) Survey 1979-80 and Pakistan Fertility Survey 1975 (PFS) birth histories were compared by urban and rural residence for each parity. Over time changes in birth spacing were marginal, reflecting only a slight increase in child spacing. The first birth interval of urban females has increased consistently by one month from 1 st parity to 4 th parity as can be seen from the

*The authors are respectively, Research Demographer and Associate Staff Economist at the Pakistan Institute of Development Economics, Islamabad. This is an abridged version of the paper read at the Fifth Annual General Meeting of the PSDE.

'For details on the methodology of constructing life tables see Smith (1980) and Hobcraft and Rodriguez (1980). 
PLM 1979-80. Urban-rural differentials reveal that urban females have shorter spacing of births as compared to rural females in both surveys (PLM 1979-80 and PFS 1975). Since it was expected that due to shorter durations of breastfeeding birth intervals may have shortened, the very marginal increase in median durations of birth interval is contrary to expectations. Evidence from the Pakistan Demographic Survey 1985-86 shows that the median of the first birth interval has further declined (18.09 months) compared to PLM (22.46 months). This may be either due to increase in age at marriage or other factors and needs further analysis.

The differences in birth spacing patterns between urban and rural women by each parity emerged quite clearly. Females in major urban $\operatorname{areas}^{2}$ had a more rapid pace of childbearing, particularly during the first and second intervals than the other two groups. In rural areas, the interval between marriage and first birth has increased. Urban females with shorter birth spacing may have shorter durations of breastfeeding, and may have higher ages at marriage. However, this seems to be consistent with the argument that urban marital fertility is higher than in rural areas.

Birth spacing by age at marriage revealed that urban females tend to have shorter median birth intervals at higher ages at marriage. Longer intervals with lower age at marriage may be attributed to subfecundity or delayed cohabitation while shorter intervals at higher ages at marriage may be attributed to a desire for rapid spacing of births in order to complete family size early. In rural areas women do not seem to be spacing their births deliberately. The longer durations with the higher age at marriage may be attributed to higher infant deaths, frequent pregnancy losses, and lower education.

Birth spacing by degree of urbanization and female education showed that the median birth interval of females in urban centres is four months shorter than those for rural females with the same education. The gap increased by 7 months in case of no female education. Thus, educated females in major urban areas have a faster tempo in achieving their family size as compared to women residing in 'other urban' and 'rural' areas. The pattern of child spacing upto the third parity is a combination of particular factors both biological and behavioural that determine family size differently in major 'urban', 'other urban' and 'rural' areas for educated females. For 4th and above intervals, educated females in major urban areas tend to have longer intervals while in other urban and rural areas educated females reflect no consistent patterns. Educated urban females having high parity (4+) have longer intervals due to greater contraceptive use and a lesser desire for additional children.

The analysis also covered regional variations in birth spacing but the patterns were not consistent. In the urban areas of Punjab and Sind relatively longer birth spacing was observed than in the rural areas but after the 2nd parity, differences disappeared. However, urban-rural differentials in all regions appear only in the 1st parity showing longer durations for rural females as compared to urban females.

The linkages between work patterns of females and birth spacing showed little variation. The median birth interval for females who had 'ever worked' were relatively shorter as compared to those who 'never worked'. It may be difficult to draw an inference from marginal differences but it shows that females engaged in economic activity desire an early completion of their family size hence a shorter birth interval.

Due to fewer cases of contraceptive users, the relationship between birth spacing and contraceptive users was not clear. However, 'ever user' category females showed shorter median intervals which may be because this small group was more educated or married late in which case the desire to complete family size soonest was quite strong.

The analysis presented in this study shows that certain associations between variables exist but cannot be interpreted in casual terms. Moreover, it is difficult to conclude that birth spacing has shown changes which may have had an impact on marital fertility.

\section{REFERENCES}

Irfan, M. (1981). "Studies in Population, Labour Force and Migration". A PIDE/ ILO UNFPA Project.

Khan, Zubeda (1985). "Breastfeeding in Pakistan" PIDE Report No. 10, Studies in Population, Labour Force and Migration Survey 1979-80.

Pakistan, Government of. Pakistan Demographic Survey (1984). Statistics Division.

Rodriguez, German, John N. Hobcraft (1980). Illustrative Analysis: Life Table Analysis of Birth Intervals in Columbia. Scientific Report No. 16 W.F.S.

Sathar, Zeba A. (1988).' "Birth Spacing in Pakistan". Journal of Biosocial Sciences. Vol. 20, No. 2

Smith, D. (1980). "Life Table Analysis". Technical Bulletin No. 6 World Fertility Survey.

${ }^{2}$ Major urban includes: Lahore, Faisalabad, Rawalpindi, Multan, Karachi and Hyderabad cities. 